\title{
Solar wind turbulence: Advances in observations and theory
}

\author{
J. J. Podesta ${ }^{1}$ \\ ${ }^{1}$ Los Alamos National Laboratory, Los Alamos, NM, 87545, USA \\ email: jpodesta@solar.stanford.edu
}

\begin{abstract}
Observations of plasma and magnetic field fluctuations in the solar wind provide a valuable source of information for the study of turbulence in collisionless astrophysical plasmas. Scientific data collected by various spacecraft over the last few decades has fueled steady progress in this field. Theoretical models, numerical simulations, and comparisons between theory and experiment have also contributed greatly to these advances. This review highlights some recent advances on the observational side including measurements of the anisotropy of inertial range fluctuations as revealed by the different scaling laws parallel and perpendicular to the mean magnetic field, measurements of the normalized cross-helicity spanning the entire inertial range which demonstrate that this quantity is scale invariant, and improved measurements of the spectrum of magnetic field fluctuations in the dissipation range that show a spectral break near the lengthscale of the electron gyro-radius. The theoretical implications of these results and comparisons between theory and observations are briefly summarized.
\end{abstract}

Keywords. Solar wind, turbulence, interplanetary medium, waves

\section{Introduction}

Turbulence is found in a wide variety of laboratory and astrophysical plasmas and MHD turbulence with high kinetic and magnetic Reynolds numbers is believed to be common in many astrophysical systems. The solar wind is one of the only such systems in which the turbulence can be measured in exquisite detail by means of spacecraft instrumentation and such measurements provide a solid foundation for developing an understanding of this fundamental physical process. The study of solar wind turbulence, both in theory and observations, has advanced steadily over the past few decades. In this review, I shall briefly summarize recent advances in three specific areas in which I have played a role including measurements of the normalized cross-helicity spectrum spanning the entire inertial range, measurements of the anisotropy of power spectra parallel and perpendicular to the mean magnetic field, and measurements of the spectrum of magnetic field fluctuations in the dissipation range.

\section{Energy spectrum and cross-helicity spectrum}

Some researchers believe that the energy spectrum of driven, steady state, homogeneous, incompressible MHD turbulence cannot be characterized by a universal power-law (Lee et al. 2010), contrary to hydrodynamic turbulence in non-conducting fluids which experimental measurements indicate has a universal scaling exponent of $5 / 3$ (Sreenivasan \& Dhruva 1998; Pope 2000). For MHD turbulence there is another school of thought that suggests there is a universal scaling with a perpendicular energy spectrum that scales like $k_{\perp}^{-3 / 2}$ when the mean magnetic field is strong, that is, when $\left(\delta B / B_{0}\right)^{2} \ll 1$, where $\delta B$ is the rms amplitude of the fluctuations at the outer scale or the largest inertial range 
scale and $B_{0}$ is the amplitude of the ambient magnetic field (Müller et al. 2003; Boldyrev 2005, 2006; Mason et al. 2006, 2008; Müller 2009; Perez \& Boldyrev 2010). In a recent study of solar wind turbulence at 1 AU, Podesta \& Borovsky (2010) have shown that the inequality $\left(\delta B / B_{0}\right)^{2} \ll 1$ is usually well satisfied and that the scaling law for the total energy (kinetic plus magnetic) is usually closer to $3 / 2$ than $5 / 3$, in agreement with these theoretical predictions. In particular, for highly Alfvénic wind, wind with high normalized cross-helicity, the spectral index for the total energy is found to be $1.540 \pm 0.033$ (Podesta \& Borovsky 2010). Highly Alfvénic wind provides the best example of MHD turbulence because it has undergone relatively little dissipation during its transit time from the sun so that, in the absence of continuous in situ forcing, its characteristics more closely resemble the turbulence generated closer to the sun. The analysis of corotating interaction regions (CIRs) has shown that large scale velocity gradients (shear) do not provide in situ forcing of solar wind turbulence near 1 AU as was previously thought because the turbulence amplitude is not observed to rise in the vicinity of the shear zone (Borovsky \& Denton 2010).

The study by Podesta \& Borovsky (2010) is the first large statistical study of energy and cross-helicity spectra spanning the entire inertial range at $1 \mathrm{AU}$. The measurements show that the total energy spectrum and the cross-helicity spectrum typically have the same spectral index and that the normalized cross-helicity $\sigma_{c}$, the ratio of the crosshelicity spectrum to the total energy spectrum, is approximately constant throughout the inertial range. That is, $\sigma_{c}$ is independent of wavenumber. Compared to previous measurements of $\sigma_{c}$ by $\mathrm{Tu}$ et al. (1989) and Marsch \& Tu (1990), the measurements by Podesta \& Borovsky (2010) increase the wavenumber range of the observations by more than one decade and show that the scale invariance of $\sigma_{c}$ covers the entire inertial range at 1 AU. This result is of significant importance for theories of solar wind turbulence and turbulence in astrophysical plasmas. It implies that the ratio of the two Elsasser spectra, the Elsasser ratio, is a constant, independent of wavenumber. This, combined with Kolmogorov's equations expressing the constancy of energy flux in $\boldsymbol{k}$-space, implies that the ratio of the energy cascade times of the two Elsasser energies $\tau^{+} / \tau^{-}$is also constant, independent of wavenumber. These ideas are often incorporated into theoretical models and now, for the first time, they have direct observational support.

\section{Anisotropy of the magnetic field spectrum}

It is well known that fluctuations in turbulent magnetized plasmas are anisotropic with respect to the direction of the local magnetic field $\boldsymbol{B}_{0}$ : the fluctuations vary much more rapidly in the direction perpendicular to $\boldsymbol{B}_{0}$ than in the direction parallel to $\boldsymbol{B}_{0}$. This has been observed in laboratory plasma experiments since the 1960s and in computer simulations since the 1970 s or 1980 s. However, it took many years before a practical turbulence phenomenology emerged that can account for this anisotropy in a simple way. The most influential phenomenological theory of this kind is the theory of incompressible MHD turbulence developed by Goldreich \& Sridhar $(1995,1997)$ which has been reviewed by Goldreich (2001) and Sridhar (2010). The theory of Goldreich \& Sridhar assumes that the eddy turnover time is approximately equal to the Alfvén wave period so that

$$
\frac{1}{k_{\perp} \delta v_{\perp}} \simeq \frac{1}{k_{\|} v_{A}}=\frac{1}{\omega_{A}} .
$$

In the inertial range, the energy flux in $k_{\perp}$-space is constant, $\left(\delta v_{\perp}\right)^{2} / \tau=\varepsilon$, and using (3.1) this implies $\left(\delta v_{\perp}\right)^{2}=\varepsilon^{2 / 3} k_{\perp}^{-2 / 3}$. Therefore, the perpendicular energy spectrum 


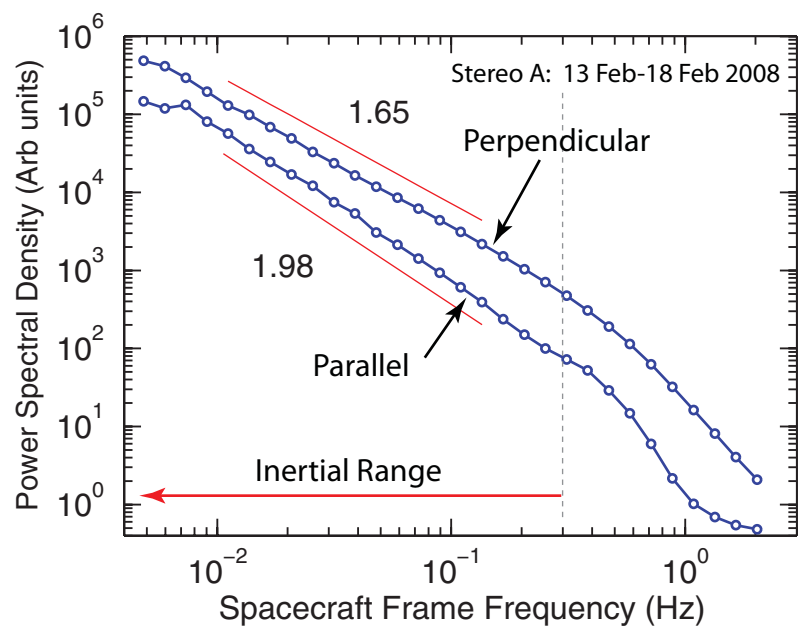

Figure 1. Example of power spectra measured perpendicular and parallel to the local mean magnetic field using wavelets (Podesta 2009). The best fit lines, in red, are offset for easier viewing and the measured inertial range slopes are 1.65 and 1.98 .

defined by $k_{\perp} E\left(k_{\perp}\right)=\left(\delta v_{\perp}\right)^{2}$ takes the form

$$
E\left(k_{\perp}\right)=C_{0} \varepsilon^{2 / 3} k_{\perp}^{-5 / 3},
$$

where $C_{0}$ is a dimensionless constant of order unity. For wavenumbers $k_{\perp}$ and $k_{\|}$related by (3.1), one may define a parallel energy spectrum such that $k_{\|} E\left(k_{\|}\right)=k_{\perp} E\left(k_{\perp}\right)$. This yields

$$
E\left(k_{\|}\right) \propto k_{\|}^{-2}
$$

Thus, in the Goldreich \& Sridhar theory the energy spectrum is proportional to $k_{\perp}^{-5 / 3}$ in the direction perpendicular to the mean field and to $k_{\perp}^{-2}$ in the direction parallel to the mean field. A different theory developed by Boldyrev (2005, 2006) predicts spectra proportional to $k_{\perp}^{-3 / 2}$ and $k_{\|}^{-2}$ in the directions perpendicular and parallel to $\boldsymbol{B}_{0}$, respectively,

Motivated by these theoretical results, it is of interest to examine solar wind fluctuations to see if the observed energy spectrum exhibits different power-law behaviors in the directions perpendicular and parallel to the mean field. The first successful measurements of this kind were performed by Horbury et al. (2008) and Podesta (2009) who found that the spectral index of magnetic field fluctuations changes from roughly $5 / 3$ for measurements perpendicular to the local mean magnetic field $\boldsymbol{B}_{0}$ to approximately 2 for measurements parallel to $\boldsymbol{B}_{0}$ (see also Podesta 2010). Horbury et al. (2008) analyzed high speed solar wind data from the Ulysses spacecraft taken above the poles of the sun at heliocentric distances near 1.4 AU and Podesta (2009) analyzed high speed streams in the ecliptic plane at $1 \mathrm{AU}$ using data from the two Stereo spacecraft. These studies have been confirmed and expanded by Luo \& Wu (2010) and by Wicks et al. (2010). An example of the anisotropy measurements is shown in Figure 1.

While the results in Figure-1 appear to agree with the scaling laws in the Goldreich \& Sridhar theory, it is premature to conclude that the Goldreich \& Sridhar scaling provides the best agreement with solar wind data. The solar wind measurements performed so far have only considered the magnetic field spectrum because of the availability of high cadence magnetic field data needed to resolve the smaller scale inertial range 
fluctuations. However, theories are generally based on the total energy spectrum - kinetic plus magnetic - which has typical spectral slopes between 1.5 and 1.6 in the solar wind and slopes closer to $3 / 2$ for highly Alfvénic wind (Podesta \& Borovsky 2010). Therefore, it is expected that measurements of the scaling laws for the total energy perpendicular and parallel to $\boldsymbol{B}_{0}$ will yield results in better agreement with the Boldyrev scaling. These measurements will be performed in the near future.

It is important to emphasize that the Goldreich \& Sridhar theory and the Boldyrev theory only apply to turbulence with vanishing cross-helicity and, therefore, these theories must be generalized to turbulence with non-vanishing cross-helicity before they can properly be applied to solar wind turbulence.

How do we measure the scaling laws in solar wind turbulence and what exactly is being measured? A single spacecraft records spatial variations of the fields along a line parallel to the average solar wind velocity. Consequently, the idea is to make measurements when the mean magnetic field $\boldsymbol{B}_{0}$ is directed either parallel or perpendicular to the flow.

The measured quantity is the mean square magnetic field $\left\langle|\boldsymbol{B}(\boldsymbol{x}+\boldsymbol{r})-\boldsymbol{B}(\boldsymbol{x})|^{2}\right\rangle$, where the displacement vector $\boldsymbol{r}$ is always parallel to the solar wind flow velocity. Therefore, when $\boldsymbol{B}_{0}$ is perpendicular to the mean flow, the displacement $\boldsymbol{r}$ is perpendicular to $\boldsymbol{B}_{0}$ and one measures the energy in the plane perpendicular to $\boldsymbol{B}_{0}$; when $\boldsymbol{B}_{0}$ is parallel to the mean flow, the displacement $\boldsymbol{r}$ is parallel to $\boldsymbol{B}_{0}$ and one measures the energy in the direction parallel to $\boldsymbol{B}_{0}$. The technique just described can also be used to make measurements at any angle $\theta$ with respect to $\boldsymbol{B}_{0}$, not just $\theta=0$ (parallel) and $\theta=\pi / 2$ (perpendicular). Note that the studies by Horbury et al. (2008) and Podesta (2009) employed wavelet analysis to effect the decomposition in time and scale, however, the analysis can also be performed using second order structure functions, as just described (Luo \& Wu 2010).

To be able to measure differences in the scaling exponents for fluctuations parallel and perpendicular to $\boldsymbol{B}_{0}$ it is important to use the local mean magnetic field (short time average) rather than the global mean magnetic field (long time average) and also to use sufficiently high cadence magnetic field data to obtain good statistics. The use of the local mean field is crucial because the dynamics of turbulent eddies with a given lengthscale $\lambda_{\perp}$ are most sensitive to the local mean magnetic field at around the same lengthscale. The necessity of using the local rather than the global mean field was shown by Cho \& Vishniac (2000) who analyzed the anisotropy of turbulent eddies in simulations of three-dimensional incompressible MHD turbulence.

Soon after the solar wind studies by Horbury et al. (2008) and Podesta (2009), an independent study based on a different analysis technique was performed by Tessein et al. (2009) who found no change in the spectral indices parallel and perpendicular to $\boldsymbol{B}_{0}$. Unfortunately, this negative result cast doubt on the previously obtained results. This negative result is partly a consequence of the fact that the study by Tessein et al. (2009) used a $1 \mathrm{hr}$ average for the mean magnetic field $\boldsymbol{B}_{0}$ (long time average) rather than the local mean magnetic field. It has since been shown that if the same analysis technique employed by Tessein et al. (2009) is applied to shorter time intervals, 7.5 min instead of $1 \mathrm{hr}$, and if higher cadence data is used, 1 sec instead of $64 \mathrm{sec}$, then the change in the spectral index from approximately $5 / 3$ in the perpendicular direction to 2 in the parallel direction is also seen using their technique (unpublished work by various groups).

Unfortunately, many other important studies cannot be discussed here due to space restrictions. For example, the work of Hnat \& Chapman (2007) and of Narita et al. (2010a, 2010b). 


\section{Spectral break and Dissipation range}

The spectrum of solar wind magnetic field fluctuations exhibits a spectral break, a steepening of the spectral slope, that marks the transition from the inertial range at large MHD scales to the dissipation range at kinetic scales (Leamon et al. 1998, 1999, Bale et al. 2005). The spectral break usually occurs around $1 / 2 \mathrm{~Hz}$ in the spacecraft frame which, by Taylor's hypothesis, is equivalent to wavenumbers of order $k_{\perp} \rho_{i} \sim 1$ or $k_{\|} d_{i} \sim 1$, where $\rho_{i}$ is the proton Larmor radius and $d_{i}=c / \omega_{p i}$ is the proton inertial length. Therefore, it should more precisely be referred to as the proton spectral break to distinguish it from the electron spectral break at higher wavenumbers. The dissipation range, sometimes called the kinetic regime or kinetic range, begins near the proton spectral break where a wide range of kinetic process come into play. These kinetic processes are believed to dissipate the turbulent energy cascade, heat the plasma, and regulate the particle distribution functions although details of the heating process are not completely understood.

Measurements of the magnetic field spectrum extending from the proton spectral break $\sim 1 / 2 \mathrm{~Hz}$ through the dissipation range to $\sim 100 \mathrm{~Hz}$ have recently been performed using the search coil magnetometers on board the four Cluster spacecraft (Saharoui et al. 2009, 2010; Alexandrova et al. 2009; Kiyani et al. 2009). With a $450 \mathrm{~Hz}$ sampling rate in burst mode, these measurements provide unprecedented time and frequency resolution for the investigation of kinetic processes and dissipation range physics. Unfortunately, however, Cluster is principally a magnetospheric mission and the four spacecraft spend relatively little time in the solar wind. Moreover, during the brief periods near apogee at $19 R_{e}$ when solar wind measurements are possible the spacecraft are close enough to the bow shock that many observed plasma kinetic effects are often caused by the bow shock and have nothing to do with processes inherent to the unobstructed solar wind (Balogh et al. 2005, Eastwood et al. 2005, Burgess et al. 2005). Whether particular intervals of "solar wind" data are devoid of bow shock or foreshock effects is difficult to determine with certainty and this is a serious concern that should be kept in mind when the data are used for solar wind science.

An example of the magnetic energy spectrum (trace spectrum) obtained from the burst mode data is shown in Figure-2. The data show that the spectrum falls off rapidly at the proton spectral break with a typical spectral slope around 3 or 4 , as already known. At higher frequencies the spectrum flattens out and for approximately one decade or more, from roughly $3 \mathrm{~Hz}$ to $30 \mathrm{~Hz}$, the spectrum is often well fit by a straight line on a log-log plot indicating a power-law behavior in this range. The spectral slope in this range is near $7 / 3$, the predicted value for a kinetic Alfvén wave (KAW) cascade and also for a whistler mode cascade, although large variations in this slope from $\sim 1.7$ to $\sim 3$ are also seen in the data-possibly caused by electron foreshock effects. Around $40 \mathrm{~Hz}$ the spectrum shows clear evidence of a high frequency spectral break first reported by Saharoui et al. (2009) and attributed to the onset of collisionless damping at wavenumbers near the electron gyroradius $k_{\perp} \rho_{e} \sim 1$ and the electron inertial length $k_{\|} d_{e} \sim 1$. Since electron physics dominates at these scales, the term electron spectral break seems appropriate.

The physical interpretation of these interesting observations is fundamental for understanding solar wind physics. Saharoui et al. (2009) interpreted the transition from a spectral slope near $5 / 3$ in the inertial range to a spectral slope near $7 / 3$ in the kinetic range as a transition from an Alfvén wave cascade to a KAW cascade at $k_{\perp} \rho_{i} \sim 1$ and they attributed the electron spectral break to Landau damping of the KAW cascade at electron scales $k_{\perp} \rho_{e} \sim 1$. This interpretation, which seemed to fit the theoretical picture elaborated by Howes et al. (2008) and Schekochihin et al. (2009), was sharply criticized by Podesta et al. (2010) who demonstrated that for typical high-speed solar wind at $1 \mathrm{AU}$ 


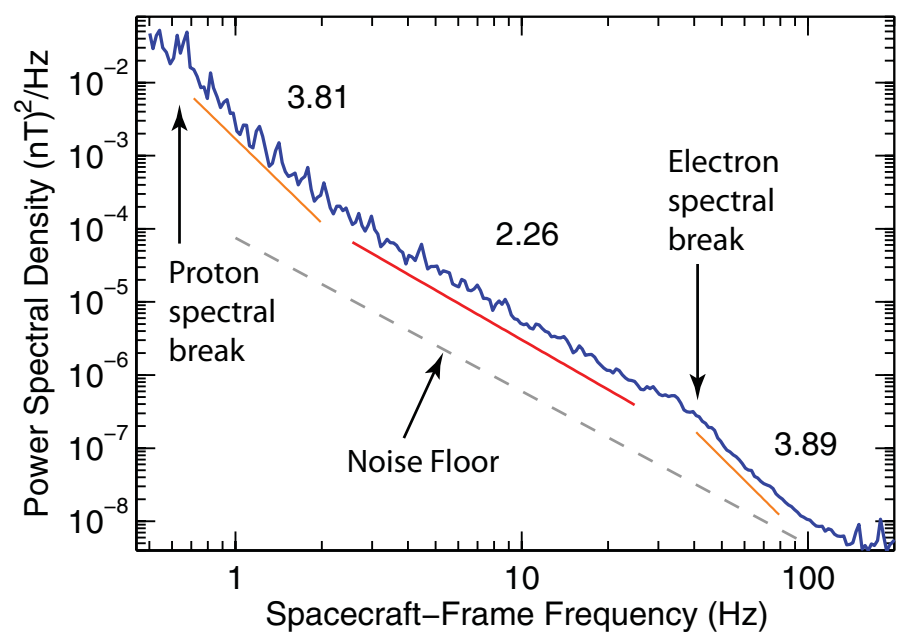

Figure 2. Example of the Cluster search coil spectrum, trace spectrum, for a $145.6 \mathrm{~s}$ interval on 19 March 2006. The line segments are the linear least squares fits obtained over different frequency ranges and have been displaced downward for easier viewing. The spectral slopes obtained from the fits are also shown.

the damping of KAWs becomes significant for $1<k_{\perp} \rho_{i}<10$ and, therefore it is likely that the KAW cascade will damp before reaching electron scales where $k_{\perp} \rho_{i} \gg 10$. This was also realized by Leamon et al. (1999). Thus, the high frequency part of the search coil spectrum, beyond approximately $5 \mathrm{~Hz}$, must be supported by some other types of wave modes - the most natural candidate being electron whistler waves as first suggested by Beinroth \& Neubauer (1981) and Denskat et al. (1983). The study by Podesta et al. (2010) indicates that the damping of KAWs should occur in a narrow range near the proton spectral break at frequencies roughly between $1 / 2 \mathrm{~Hz}$ and $4 \mathrm{~Hz}$ in the spacecraft frame, where the most rapid decrease in the observed spectrum occurs. That is where the dominant dissipation of solar wind turbulence is believed to occur. Leamon et al. (1999) reached similar conclusions using a different theoretical model.

In light of the study by Podesta et al. (2010), Saharoui et al. (2010) in their second Physical Review Letter changed the physical interpretation given in Saharoui et al. (2009) by emphasizing the significant Landau damping of KAWs that is expected to occur in a narrow range near the proton spectral break $k_{\perp} \rho_{i} \sim 1$ and by showing that this coincides with the steep drop in the spectrum at that point. Saharoui et al. (2010) also made a significant contribution to the subject by using the wave telescope technique to determine the observed dispersion relation of the waves as a function of the propagation angle in the wavenumber range $0.1<k_{\perp} \rho_{i}<2$. The observations were shown to be in reasonable agreement with the linear Vlasov-Maxwell dispersion relation for KAWs. The analysis also showed that the propagation direction of the waves was nearly perpendicular to the mean magnetic field $B_{0}$ as predicted by anisotropic turbulence phenomenologies. Thus, Saharoui et al. (2010) provided new evidence to corroborate that KAWs are the energetically dominant wave mode in the vicinity of the proton spectral break and that the damping of these waves is the dominant dissipation mechanism for solar wind turbulence.

\section{References}

Alexandrova, O., Saur, J., Lacombe, C., Mangeney, A., Mitchell, J., Schwartz, S. J., \& Robert, P. 2009, Phys. Rev. Lett., 103, 165003 
Bale, S. D., Kellogg, P. J., Mozer, F. S., Horbury, T. S., \& Reme, H. 2005, Phys. Rev. Lett., 94, 215002

Balogh, A., et al. 2005, Space Sci. Rev., 118, 155

Beinroth, H. J., \& Neubauer, F. M. 1981, J. Geophys. Res., 86, 7755

Boldyrev, S. 2005, Astrophys. J., 626, L37

-. 2006, Phys. Rev. Lett., 96, 115002

Borovsky, J. E., \& Denton, M. H. 2010, J. Geophys. Res. (Space Physics), 115, A10101

Burgess, D., et al. 2005, Space Sci. Rev., 118, 205

Cho, J., \& Vishniac, E. T. 2000, Astrophys. J., 539, 273

Denskat, K. U., Beinroth, H. J., \& Neubauer, F. M. 1983, J. Geophysics Zeitschrift Geophysik, 54,60

Eastwood, J. P., Lucek, E. A., Mazelle, C., Meziane, K., Narita, Y., Pickett, J., \& Treumann, R. A. 2005, Space Sci. Rev., 118, 41

Goldreich, P. 2001, Astrophys. Space Sci., 278, 17

Goldreich, P., \& Sridhar, S. 1995, Astrophys. J., 438, 763

-. 1997, Astrophys. J., 485, 680

Horbury, T. S., Forman, M., \& Oughton, S. 2008, Phys. Rev. Lett., 101, 175005

Howes, G. G., Cowley, S. C., Dorland, W., Hammett, G. W., Quataert, E., \& Schekochihin, A. A. 2008, J. Geophys. Res., 113, 5103

Kiyani, K. H., Chapman, S. C., Khotyaintsev, Y. V., Dunlop, M. W., \& Sahraoui, F. 2009, Phys. Rev. Lett., 103, 075006

Leamon, R. J., Smith, C. W., Ness, N. F., Matthaeus, W. H., \& Wong, H. K. 1998, J. Geophys. Res., 103, 4775

Leamon, R. J., Smith, C. W., Ness, N. F., \& Wong, H. K. 1999, J. Geophys. Res., 104, 22331

Lee, E., Brachet, M. E., Pouquet, A., Mininni, P. D., \& Rosenberg, D. 2010, Phys. Rev. E, 81, 016318

Luo, Q. Y., \& Wu, D. J. 2010, Astrophys. J., 714, L138

Mason, J., Cattaneo, F., \& Boldyrev, S. 2006, Phys. Rev. Lett., 97, 255002

-. 2008, Phys. Rev. E., 77, 036403

Müller, W. 2009, in Lecture Notes in Physics, Berlin Springer Verlag, Vol. 756, Interdisciplinary Aspects of Turbulence, ed. W. Hillebrandt \& F. Kupka, 223-254

Müller, W.-C., Biskamp, D., \& Grappin, R. 2003, Phys. Rev. E., 67, 066302

Narita, Y., Glassmeier, K., Sahraoui, F., \& Goldstein, M. L. 2010a, Phys. Rev. Lett., 104, 171101

Narita, Y., Sahraoui, F., Goldstein, M. L., \& Glassmeier, K. 2010b, J. Geophys. Res. A, 115, 4101

Perez, J. C. \& Boldyrev, S. 2010a, Astrophys. J., 710, L63

-. 2010b, Phys. Plasmas, 17, 055903

Podesta, J. J. 2009, Astrophys. J., 698, 986

Podesta, J. J. 2010, in AIP Conference Series, Vol. 1216, Twelfth International Solar Wind Conference, ed. M. Maksimovic, K. Issautier, N. Meyer-Vernet, M. Moncuquet \& F. Pantellini, $128-131$

Podesta, J. J. \& Borovsky, J. E. 2010, Phys. Plasmas (in press)

Podesta, J. J., Borovsky, J. E., \& Gary, S. P. 2010, Astrophys. J., 712, 685

Pope, S. B. 2000, Turbulent Flows (Cambridge University Press)

Sahraoui, F., Goldstein, M. L., Belmont, G., Canu, P., \& Rezeau, L. 2010, Phys. Rev. Lett., 105, 131101

Sahraoui, F., Goldstein, M. L., Robert, P., \& Khotyaintsev, Y. V. 2009, Phys. Rev. Lett., 102, 231102

Schekochihin, A. A., Cowley, S. C., Dorland, W., Hammett, G. W., Howes, G. G., Quataert, E., \& Tatsuno, T. 2009, Astrophys. J. Suppl., 182, 310

Sreenivasan, K. R., \& Dhruva, B. 1998, Progress of Theoretical Physics Supplement, 130, 103

Tessein, J. A., Smith, C. W., MacBride, B. T., Matthaeus, W. H., Forman, M. A., \& Borovsky, J. E. 2009, Astrophys. J., 692, 684

Wicks, R. T., Horbury, T. S., Chen, C. H. K., \& Schekochihin, A. A. 2010, Mon. Not. R. Astron. Soc., 407, L31 